\title{
Control over emissivity of zero-static-power thermal emitters based on phase-changing material GST
}

\begin{abstract}
Kai-Kai Du ${ }^{1}$, Qiang Li ${ }^{1}$, Yan-Biao Lyu², Ji-Chao Ding ${ }^{1}$, Yue Lu ${ }^{1}$, Zhi-Yuan Cheng ${ }^{2}$ and Min Qiu ${ }^{1}$
Controlling the emissivity of a thermal emitter has attracted growing interest, with a view toward a new generation of thermal emission devices. To date, all demonstrations have involved using sustained external electric or thermal consumption to maintain a desired emissivity. In the present study, we demonstrated control over the emissivity of a thermal emitter consisting of a film of phase-changing material $\mathrm{Ge}_{2} \mathrm{Sb}_{2} \mathrm{Te}_{5}$ (GST) on top of a metal film. This thermal emitter achieves broad wavelength-selective spectral emissivity in the mid-infrared. The peak emissivity approaches the ideal blackbody maximum, and a maximum extinction ratio of $>10 \mathrm{~dB}$ is attainable by switching the GST between the crystalline and amorphous phases. By controlling the intermediate phases, the emissivity can be continuously tuned. This switchable, tunable, wavelength-selective and thermally stable thermal emitter will pave the way toward the ultimate control of thermal emissivity in the field of fundamental science as well as for energy harvesting and thermal control applications, including thermophotovoltaics, light sources, infrared imaging and radiative coolers.
\end{abstract} Light: Science \& Applications (2017) 6, e16194; doi:10.1038/lsa.2016.194; published online 27 January 2017

Keywords: absorptivity; emissivity; mid-infrared; switchable; thermal emitters

\section{INTRODUCTION}

Any object with a temperature above absolute zero gives off light or thermal emission. Black soot generally shows high emissivity, whereas smooth metal exhibits low emissivity. Other natural materials are characterized by their own emissivities. The intrinsic thermal emission of these materials is usually spectrally broad, and their emissivities cannot be easily tuned. However, in certain applications, such as biochemical sensing ${ }^{1}$, light sources ${ }^{2,3}$ and thermal emitters with tailored emissivities are highly desirable. For instance, a sharp cutoff in emission wavelength is needed in thermophotovoltaics ${ }^{4-8}$; passive radiative coolers demand that the emitters radiate only in the spectral range of $8-13 \mu \mathrm{m}^{9-11}$. In addition, switchable and tunable thermal emission is necessary in applications such as infrared camouflage $^{12}$ and heat management ${ }^{13-15}$.

Conventional thermal emission with spectral selectivity is based on micro/nano-structures such as gratings ${ }^{16}$, photonic crystals ${ }^{17}$, photonic cavities $^{18-20}$, nanoantennas ${ }^{21}$ and metamaterials ${ }^{22}$. By altering the structural parameters of the micro/nano-structures with advanced micro/nano-fabrication, wavelength-selective thermal emitters from $\mathrm{THz}$ to visible can be realized ${ }^{16-30}$. However, static micro/nanostructures do not offer flexible tunability in terms of thermal emissivity. To date, several strategies have been implemented to engineer control over the thermal emissivity. (i) Electrically tunable materials (such as quantum wells and graphene) have been employed for modulation of thermal emissivity. By electrically tuning intersubband absorption in n-type quantum wells, the emissivity of a narrowband mid-infrared thermal emitter based on a photonic crystal slab can be tailored statically $y^{31,32}$ or dynamically ${ }^{33-35}$. Using an electrostatic gate to control the charge density in graphene, modulation of the emissivity of a narrow-band mid-infrared thermal emitter composed of graphene/SiNx/Au nanoresonators by $3 \%$ has been achieved ${ }^{36}$. However, sustained electric consumption is needed to maintain a certain state of the tunable materials. (ii) Materials with high thermal expansion coefficients (such as $\mathrm{SiNx}$ ) have been introduced in microelectro-mechanical-system-based emitters ${ }^{37}$. By manipulating the distance between the top metamaterial pattern and the bottom metallic film by applying heat, control of the temperature-dependent emissivity is achieved. However, the emissivity difference is lower than $35 \%$, and sustained thermal consumption is needed to maintain the mechanical distance so as to control the emissivity. (iii) Phase-changing materials (PCMs) have also been introduced to modulate the thermal emissivity owing to their different optical and infrared properties in their different phases. VOx is a typical PCM and exhibits an insulator-tometal phase transition at a temperature of $67^{\circ} \mathrm{C}^{12}$. By controlling the phases of VOx at different temperatures, a tunable thermal emissivity for an emitter composed of a VOx layer on a sapphire substrate has been demonstrated ${ }^{12}$. However, to preserve the metal phase of VOx, the temperature of the thermal emitter needs to be maintained above $67^{\circ} \mathrm{C}$. Otherwise, a metal-to-insulator phase recovery is activated. Although significant progress has been made to control the thermal emissivity, there has yet to emerge an energy-efficient solution to maintaining a desired emissivity with zero static power consumption at room temperature.

${ }^{1}$ State Key Laboratory of Modern Optical Instrumentation, College of Optical Science and Engineering, Zhejiang University, Hangzhou 310027, China and ${ }^{2}$ College of Information Science \& Electronic Engineering, Zhejiang University, Hangzhou 310027, China

Correspondence: Q Li, Email: qiangli@zju.edu.cn

Received 14 April 2016; revised 18 July 2016; accepted 20 July 2016; accepted article preview online 22 July 2016 
$\mathrm{Ge}_{2} \mathrm{Sb}_{2} \mathrm{Te}_{5}$ (GST) is another PCM material with amorphous and crystalline phases $^{38-45}$. It has been widely used as a commercialized rewriteable optical storage medium in digital video disks owing to its phase-changing performance ${ }^{46,47}$. The atom distributions of GST in the amorphous and crystalline phases are shown in Figure $1 \mathrm{a}^{48}$. Although the atoms are chaotically arranged in the amorphous phase, they are aligned in an orderly manner in the crystalline phase, leading to distinct infrared properties. The GST film can be fabricated by magnetron sputtering, and the as-deposited GST film is initially in the amorphous phase. The amorphous GST (termed as 'aGST') transforms into the crystalline phase (termed as 'cGST') when it is annealed above $160^{\circ} \mathrm{C}^{49}$. For reamorphization, a quick annealing process over $640^{\circ} \mathrm{C}$ must be applied ${ }^{50}$. Once the crystallization or reamorphization has been accomplished, the GST film remains stable in a certain phase even for years at room temperature unless the phase transition conditions are satisfied, making it a very appealing material for energy-efficient optoelectronic devices with zero static power. Based on these unique properties, GST has been applied in various energyefficient switchable photonic devices such as absorbers ${ }^{39}$, multi-level memory $^{51}$, chiral metamaterials ${ }^{40}$ and color devices ${ }^{41,52,53}$.

In the present study, we demonstrated control over the emissivity of a zero-static-power mid-infrared thermal emitter based on a GST film on top of a metal film. The emissivity of this thermal emitter is switchable, tunable and wavelength-selective. The wavelengthselectivity is accomplished by altering the GST thickness. The switchable thermal emission is achieved by switching the GST between amorphous and crystalline phases. By controlling the intermediate phases composed of different proportions of amorphous and crystalline molecules of the GST, the emissivity of the thermal emitter can be continuously tuned. This GST-based thermal emitter presents several distinctive advantages. (i) The two phases (amorphous and crystalline) of the GST show distinct infrared properties, resulting in a sharp contrast between on and off states in the switchable emissivity. When the GST is in the crystalline phase, an emissivity approaching the ideal blackbody maximum (on state) is realized, and the emissivity is significantly suppressed to below 0.2 (off state) in the amorphous phase. By switching between the amorphous and crystalline phases of the GST, a maximum extinction ratio of up to $11 \mathrm{~dB}$ can be realized. (ii) The transition between the amorphous and crystalline phases of the GST reveals hybrid phase behaviors, which can be exploited to implement tunable emission by properly controlling the annealing time and the annealing temperature. (iii) Wavelength-selective thermal emission in a broad range (from 3 to $15 \mu \mathrm{m}$ ) can also be enabled by varying the GST thickness. (iv) The fabrication of this thermal emitter involves only simple film deposition; therefore, this thermal emitter benefits from the virtue of large-area and lithography-free fabrication as well as design flexibility.

\section{MATERIALS AND METHODS}

\section{Fabrication of the GST-Au samples}

The GST-Au samples are fabricated starting with a 120-nm-thick gold film deposition. Because the gold film is sufficiently optically thick, there is low demand for the substrate. The gold film is deposited on the BK7 glass substrate via a thermal evaporation technique. The GST layer is then deposited on the gold film by magnetron sputtering, in which germanium is DC sputtered and the other two components (stibium and tellurium) are radio frequency (RF) sputtered with a 2:2:5 deposition rate ratio. The as-deposited GST alloy is in the amorphous phase, and a $1.5 \mathrm{~min}$ annealing process at $180^{\circ} \mathrm{C}$ on a hot plate is performed to obtain the crystalline GST in this paper.

\section{Absorptivity measurements}

The absorptivities of the GST-Au samples are derived by measuring the reflectivities. Because the gold film is sufficiently optically thick, transmission does not exist in the experiment. Thus, the absorptivity (A) and the reflectivity $(R)$ satisfy $A=1-R$. The vertical reflectivities are measured with the Bruker Vertex 70 FTIR and the Hyperion 1000 infrared microscope equipped with a liquid nitrogen refrigerated MCT
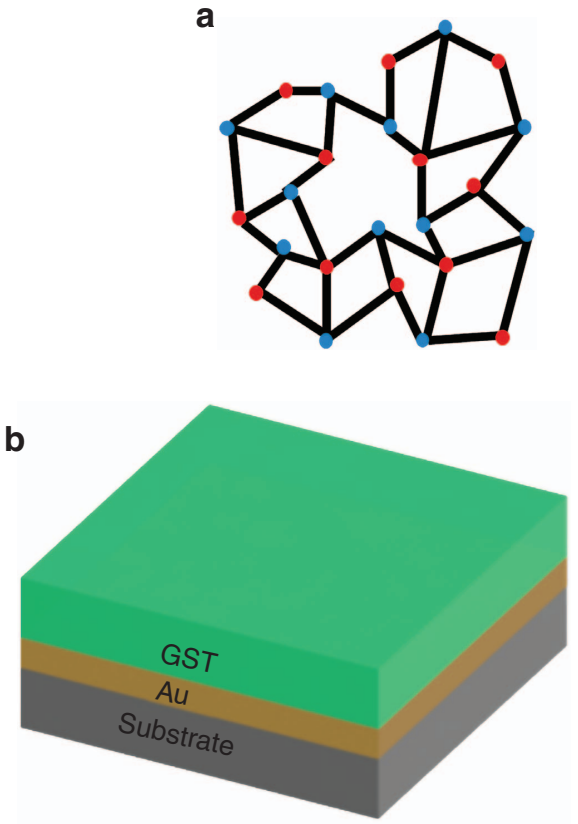

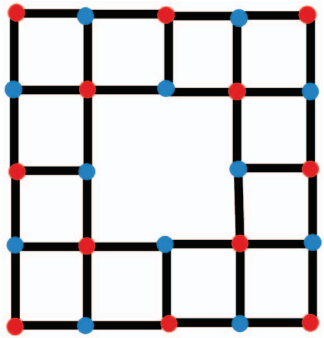

C

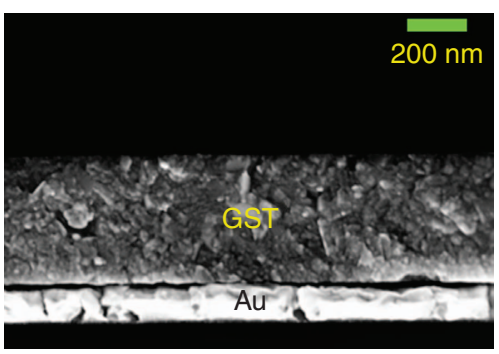

Figure 1 (a) Atom distribution diagrams of the two phases (amorphous and crystalline) of the GST. The red and blue dots denote the Ge/Sb atoms and Te atoms, respectively. (b) A 3D schematic of the switchable and tunable thermal emitter composed of a GST film on top of a gold film. (c) An SEM image of a cross-section of the fabricated thermal emitter. 
detector, and the oblique reflectivities are measured with a DTGS detector on the Vertex 70 FTIR equipped with the A513 attachment.

\section{Emissivity measurements}

The emitted spectra of the black soot and the GST-Au samples are measured by the Bruker Vertex 70 FTIR equipped with a DTGS detector. The samples are fixed on a temperature controller, and the emitted power is sent into the FTIR and detected by the DTGS detector. The temperatures of both the black soot and the GST-Au samples are controlled over $100^{\circ} \mathrm{C}$ to ensure high emitted power and to decrease the noise. Every measurement is repeated 16 times to further reduce the noise. The infrared photographs are taken by the FLIR TG165 infrared camera.

\section{RESULTS AND DISCUSSION}

A schematic diagram of the switchable and tunable thermal emitter is shown in Figure 1b. A GST layer is deposited on a gold film (also termed as 'GST-Au sample'). The gold film, which is sufficiently optically thick, can be replaced by other metal (silver, aluminum and so on) films. Figure 1c presents an SEM image of a cross-section of the device with a 450 -nm-thick GST on top of a 120 -nm-thick gold layer.

According to Kirchhoff's law of thermal radiation, the absorptivity of an object is equal to its thermal emissivity. Before determining the emissivity of the thermal emitter, its absorption properties as an absorber are studied first. The electromagnetic responses of the GST-Au samples are simulated by COMSOL Multiphysics software. In the simulation, the relative permittivity of gold is derived from Rakić's work ${ }^{54}$. The relative permittivity of the GST (shown in Supplementary Fig. S1) is determined based on the spectrophotometric method from the transmission and reflection measurements ${ }^{55}$. From the measured permittivity, it can be concluded that the GST in the amorphous phase is transparent in the mid-infrared, whereas the GST in the crystalline phase is highly absorptive.

The absorptivities of the GST-Au samples at normal incidence are investigated via simulations and experiments, which are presented in Figure $2 \mathrm{a}$ and $2 \mathrm{~b}$, respectively. The absorptivities of the samples at oblique incidence are presented in Supplementary Fig. S2. In the midinfrared, the metal layer can be approximately regarded as a perfect electric conductor (PEC) owing to the large real and imaginary parts of its refractive index. Thus, nearly complete reflection with a phase shift of $\pi$ at the GST-Au interface is formed. Without the metal layer, fundamental resonance can also occur at a GST thickness of approximately $\lambda / 2 n$ ( $n$ is the real part of the refractive index of GST and $\lambda$ represents the resonant wavelength); however, the maximum

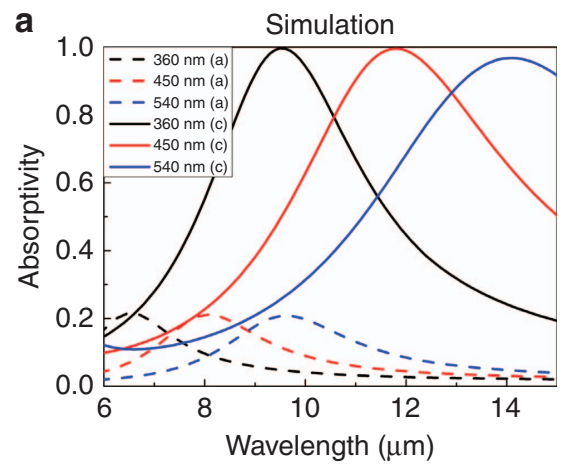

absorption is limited to $50 \%^{56,57}$ (Supplementary Fig. S3). When a gold film is placed below the GST film, not only is the GST thickness halved but also a nearly unity absorptivity can be achieved for the cGST-Au sample (see Section 3 of the Supplementary Information for more details). Because the aGST is nearly transparent, a resonant cavity is formed at a GST thickness of approximately $\lambda / 4 n$ such that a round trip inside the cavity could accumulate a 0 (or $2 \pi$ ) phase shift ${ }^{58}$. At resonance, absorption and field penetration can be observed in the metal film in both the aGST-Au and cGST-Au samples because the metal film is not an ideal PEC. For the aGST-Au sample, this small absorption and field penetration in the metal film contribute to the total absorption (below 20\%) at resonance; for the cGST-Au sample, nearly unity absorption arises from resonance-induced field enhancement in the highly absorptive cGST layer. Therefore, a sharp contrast between the absorptivities of the aGST-Au and cGST-Au samples is created. By increasing the GST thickness from 360 to $540 \mathrm{~nm}$, the peak absorption wavelength shifts approximately linearly from 9 to $13 \mu \mathrm{m}$, demonstrating the wavelength-selectivity of the device. In the simulation, a broad wavelength selectivity from 3 to $15 \mu \mathrm{m}$ wavelength can be achieved (the permittivity of the GST beyond a wavelength of $15 \mu \mathrm{m}$ is unknown). Excellent agreement is obtained between the experimental data and the simulations. The slight difference can be attributed to light scattering loss induced by rough film surfaces.

To unveil the physics behind the sharp contrast between absorptivities of the aGST-Au and cGST-Au samples, the specific absorptivity in each layer (GST layer and metal layer) is further investigated (Figure $3 \mathrm{a}$ and $3 \mathrm{~b}$ ). Meanwhile, normalized electric field $(|E|)$ and resistive loss $(Q)$ distributions at resonant wavelengths for the GST-Au samples are presented in Figure $3 \mathrm{c}$ and $3 \mathrm{~d}$, respectively. It is obvious that the electric field intensity in the device decreases as light propagates into the GST layers and is quite weak at the GST-Au interface (Figure $3 \mathrm{c}$ ), demonstrating the $\pi$-phase shift due to the reflection in metal and the $\lambda / 4 n$-thick GST layer. The absorption of light leads to heat generation, which can be characterized by a resistive loss (also termed as 'heat power volume density'). The resistive loss $Q$ is related to the imaginary part of the material relative permittivity $\left(\varepsilon^{\prime \prime}\right)$ and the intensity of the electric field $(E)$ by $Q=\pi c \varepsilon_{0} \varepsilon^{\prime \prime}(\lambda)|E|^{2} / \lambda$, where $c$ is the speed of light in vacuum and $\varepsilon_{0}$ is the vacuum permittivity ${ }^{59}$. Because of the transparent property of the aGST, there are no resistive losses in the aGST layer even though its electric field is much stronger than that of the cGST layer. By comparison, large resistive losses occur in the cGST layers for the cGST-Au samples. The highly absorptive GST combined with resonant field enhancement contributes to nearly perfect absorption in the cGST-Au samples. In both cases, there are

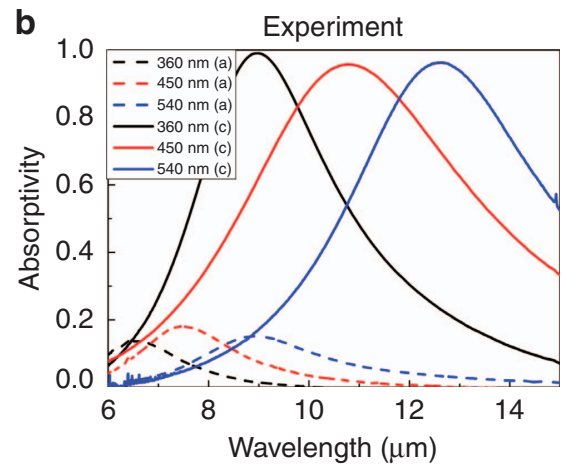

Figure 2 (a, b) Simulated and experimental normal-incident absorptivity of the cGST-Au and aGST-Au samples in the mid-infrared with three different GST thicknesses (360, 450 and $540 \mathrm{~nm}$ ). The dashed and solid lines are for the aGST-Au and cGST-Au samples, respectively. (a) and (c) denote the aGST-Au and cGST-Au samples, respectively. 
resistive losses in the top surfaces of the metal layers due to the noninfinite imaginary part of its relative permittivity and residual electric field penetration into the metal.

The emitted spectra are measured by a Fourier transform infrared spectrometer (FTIR) equipped with a room temperature-doped triglycine sulfate (DTGS) detector. Thermal emission from the central areas (denoted by the red dotted circles in Figure 4a) of the samples is sent into the FTIR for detection. Black soot is conventionally regarded as a perfect reference owing to its high wavelength-independent emissivity. Here the black soot reference is produced by firing a rectangular stainless steel slice with a candle, and its emissivity $\left(\varepsilon_{\text {ref }}(\lambda, T)\right.$, where $\lambda$ is the wavelength and $T$ is the temperature) is assumed to be 0.97 . With the measured emission spectra of the GST-Au samples $\left(S_{\text {sample }}(\lambda, T)\right)$ and the black-soot reference $\left(S_{\text {ref }}(\lambda, T)\right)$ (shown in Supplementary Fig. S4a), the normalized emissivities $\left(\varepsilon_{\text {sample }}(\lambda, T)\right)$ of the GST-Au samples can be derived from ${ }^{12}$

$$
\varepsilon_{\text {sample }}(\lambda, T)=\frac{S_{\text {sample }}(\lambda, T)}{S_{\text {ref }}(\lambda, T)} \varepsilon_{\text {ref }}(\lambda, T)
$$

The derived emissivities of the GST-Au emitters with different GST thicknesses at $100{ }^{\circ} \mathrm{C}$ are presented in Figure $4 \mathrm{~b}$. The cGST-Au emitter yields a notably high peak emissivity approaching the ideal blackbody maximum. The peak emissivities are $0.92,0.95$ and 0.97 for GST thicknesses of 360, 450 and $540 \mathrm{~nm}$, respectively. The emissivities of the aGST-Au emitters are low, and the emission peaks are below 0.20. Therefore, this thermal emitter shows two distinct states: an on state with a high emissivity when the GST is in the crystalline phase and an off state with a low emissivity when the GST is in the amorphous phase. The extinction ratios and the differential emissivities between the on and off states of the GST-Au emitters are presented in
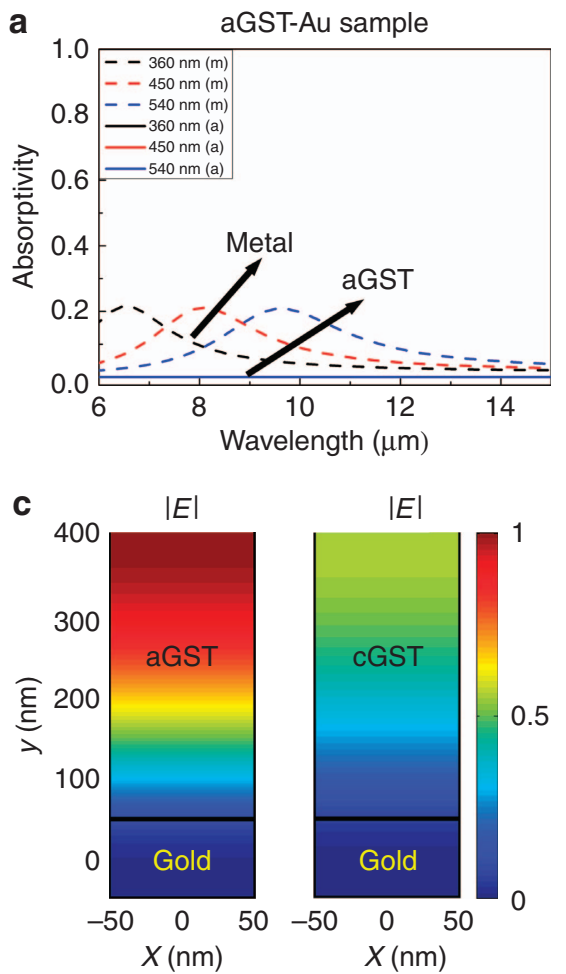

Figure $4 \mathrm{c}$ and Supplementary Fig. S4b, respectively. A maximum extinction ratio of 10.15, 11.04 and $10.98 \mathrm{~dB}$ can be obtained for GST thicknesses of 360, 450 and $540 \mathrm{~nm}$, respectively. The peak emission wavelength shifts further with increasing cGST film thickness, thereby showing promise as a wavelength-selective thermal emitter. Although the emissivity is measured directly, whereas the absorptivity is characterized through transmission and reflection, excellent agreement between the experimental absorptivity (Figure 2b) and emissivity (Figure $4 \mathrm{~b}$ ) can be observed, verifying Kirchhoff's law of thermal radiation.

The visible and infrared photographs of the black soot, the aGST$\mathrm{Au}$ and cGST-Au emitters at $100^{\circ} \mathrm{C}$ are presented in Figure 5a. The temperature label of the infrared camera is based on the integration of received power from 8 to $14 \mu \mathrm{m}$. In the infrared photographs, it is obvious that the black soot shows strong emission, whereas the aGSTAu emitter exhibits weak emission. To reveal angular dependences of emission, the oblique views of the infrared photographs of the samples are presented in Supplementary Fig. S5. The angular-dependent emitted power (from 8 to $14 \mu \mathrm{m}$ ) of the black soot and the cGST$\mathrm{Au}$ emitter can be derived from the displayed temperature on the infrared camera. The normalized angular-dependent emitted power of the cGST-Au emitter with respect to the normally emitted power of the black soot is provided in Figure 5b. As the angle increases, the thermal emission of both the black soot and the cGST-Au emitter decreases slowly, indicating that the thermal emission is robust with respect to the emission angle.

So far, we have mainly focused on the switchable and wavelengthselective properties of the GST-Au emitters. The phase transition of the GST between the amorphous phase and the crystalline phase is
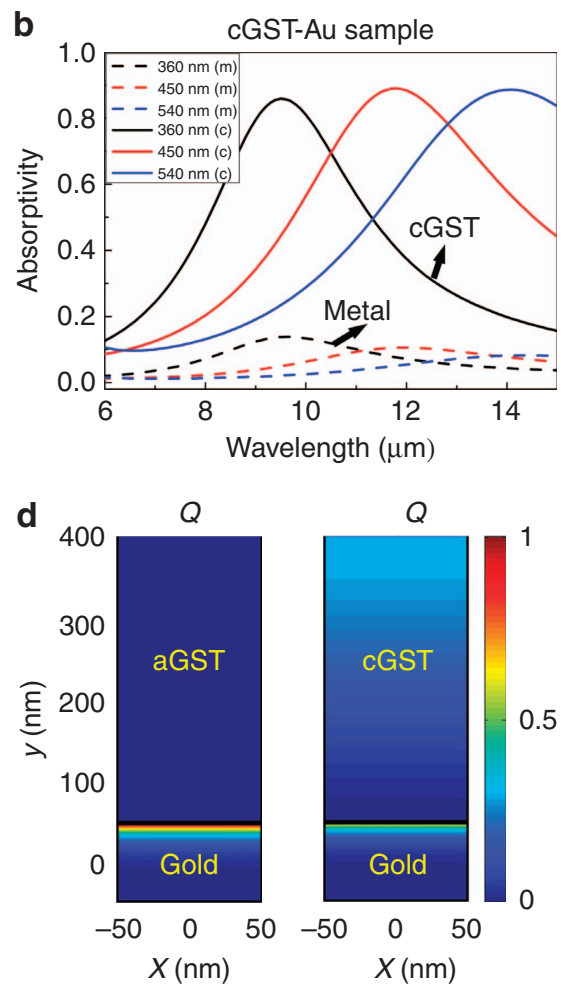

Figure 3 (a, b) Simulated absorptivity versus wavelength in specific layers (GST layer and metal layer) for the aGST-Au sample and the cGST-Au sample with different GST thicknesses $(360,450$ and $540 \mathrm{~nm}$ ). $\mathrm{m}$, a and c denote the metal layer, the aGST layer and the cGST layer, respectively. Simulated normalized resonant (c) electric field $I E$ and (d) resistive loss $Q$ distribution in the GST-Au samples with a 360-nm-thick GST film. The resonant wavelengths are 6.5 and $9.5 \mu \mathrm{m}$ for the aGST-Au and cGST-Au samples, respectively. 

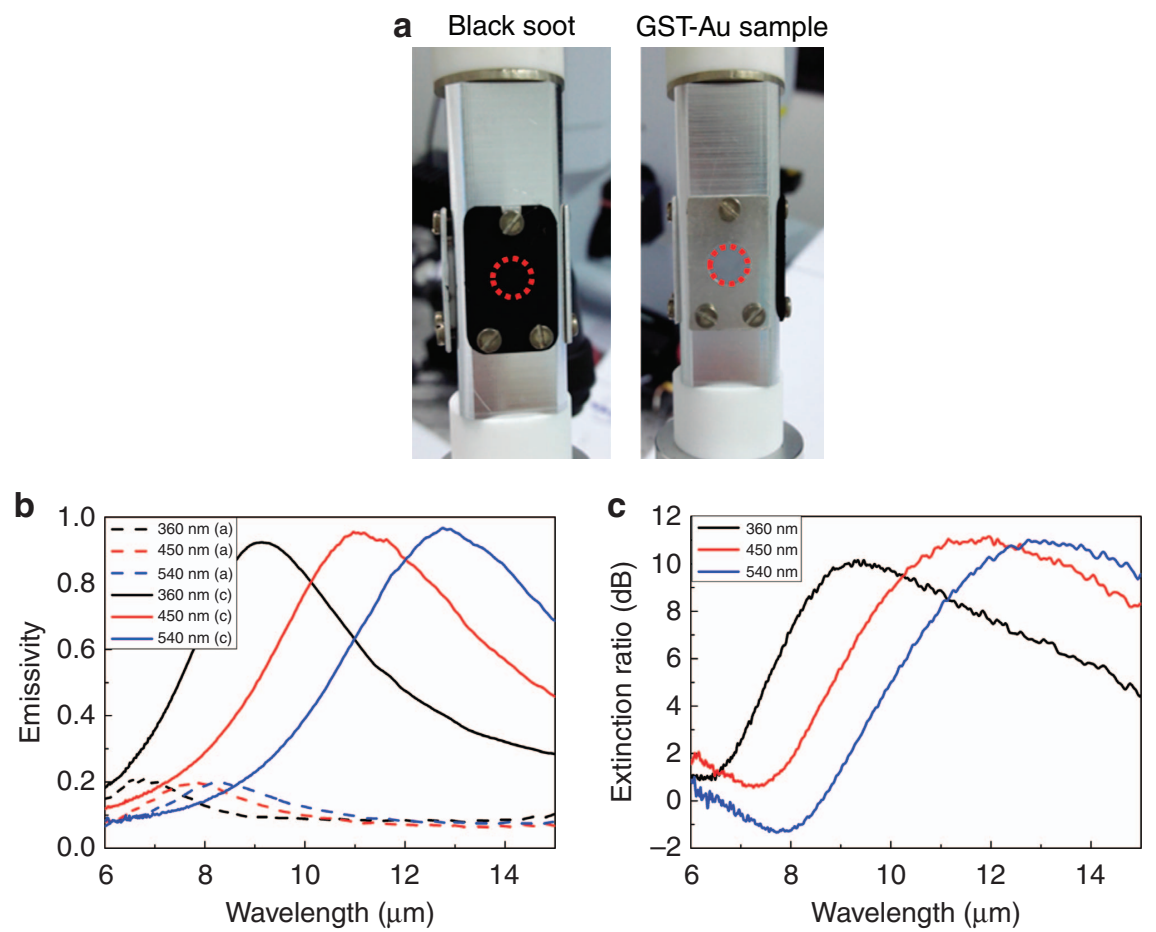

Figure 4 (a) Experimental photographs of the black soot (left) and the GST-Au emitter (right) on a temperature controller. (b) Thermal emissivity curves of GST-Au emitters with different GST thicknesses $\left(360,450\right.$ and $540 \mathrm{~nm}$ ) at $100{ }^{\circ} \mathrm{C}$. (c) Extinction ratio (in dB) between the on and off states of the GST-Au emitter with different GST thicknesses at $100{ }^{\circ} \mathrm{C}$
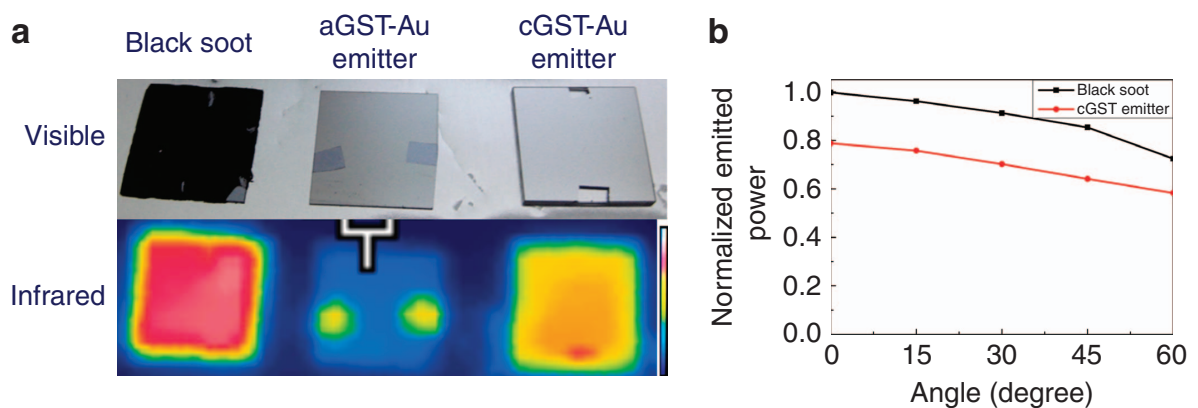

Figure 5 (a) Visible and infrared photographs of the black soot (left), the aGST-Au emitter (middle) and the cGST-Au emitter (right) at $100{ }^{\circ} \mathrm{C}$. (b) Measured emitted power from $0^{\circ}$ to $60^{\circ}$ for the black soot and the cGST-Au emitter. The GST thickness is $540 \mathrm{~nm}$. The power is normalized to the emitted power of the black soot at $0^{\circ}$.

gradual rather than abrupt. During this phase transition, the GST film can be assumed to be at intermediate phases composed of different proportions of amorphous and crystalline molecules. By controlling different intermediate phases of the GST, a tunable thermal emitter can be realized. This tunable property can be investigated by gradually increasing the sample temperature and simultaneously extracting the thermal emission spectrum. When the temperature is raised from 140 to $170{ }^{\circ} \mathrm{C}$ in $30 \mathrm{~min}$ with a step of $1{ }^{\circ} \mathrm{C}$, the emissivity curves of the GST-Au emitter with a GST thickness of $450 \mathrm{~nm}$ are recorded and presented in Figure 6a. The peak emissivity increases with temperature and rises sharply between 150 and $155^{\circ} \mathrm{C}$. The peak emissivity approaches the ideal blackbody maximum at $170^{\circ} \mathrm{C}$. The temperature-dependent emissivities at different wavelengths are explicitly presented in Figure 6b, showing steep upslopes between 150 and $155^{\circ} \mathrm{C}$ except for wavelengths of 7 and $8 \mu \mathrm{m}$. The temperaturedependent emissivity differences and extinction ratios in $\mathrm{dB}$ between the intermediate GST-Au samples and the aGST-Au sample are provided in Supplementary Fig. S6. The relative refractive indices of the GST during the intermediate phases are measured and presented in Figure $6 c$. Both the real and imaginary parts of the refractive index increase with temperature. The increase in the real part is primarily responsible for the resonance shift to longer wavelength, and the increase in the imaginary part contributes to the increase in the emissivity. The intermediate phases of the GST are also stable at room temperature, and therefore no sustained external thermal consumption is needed to maintain a desired emissivity for the GST-Au emitter. The intermediate phase of the GST is dominated by both annealing time and annealing temperature (Supplementary Fig. S7). By controlling the annealing process, the crystallization level and the intermediate phase of the GST can be correspondingly tailored, thus providing an avenue for tunable thermal emission.

In this paper, the crystallization process of the GST is investigated by a thermal annealing method, while reamorphization (phase transition from the crystalline phase back to the amorphous phase) 

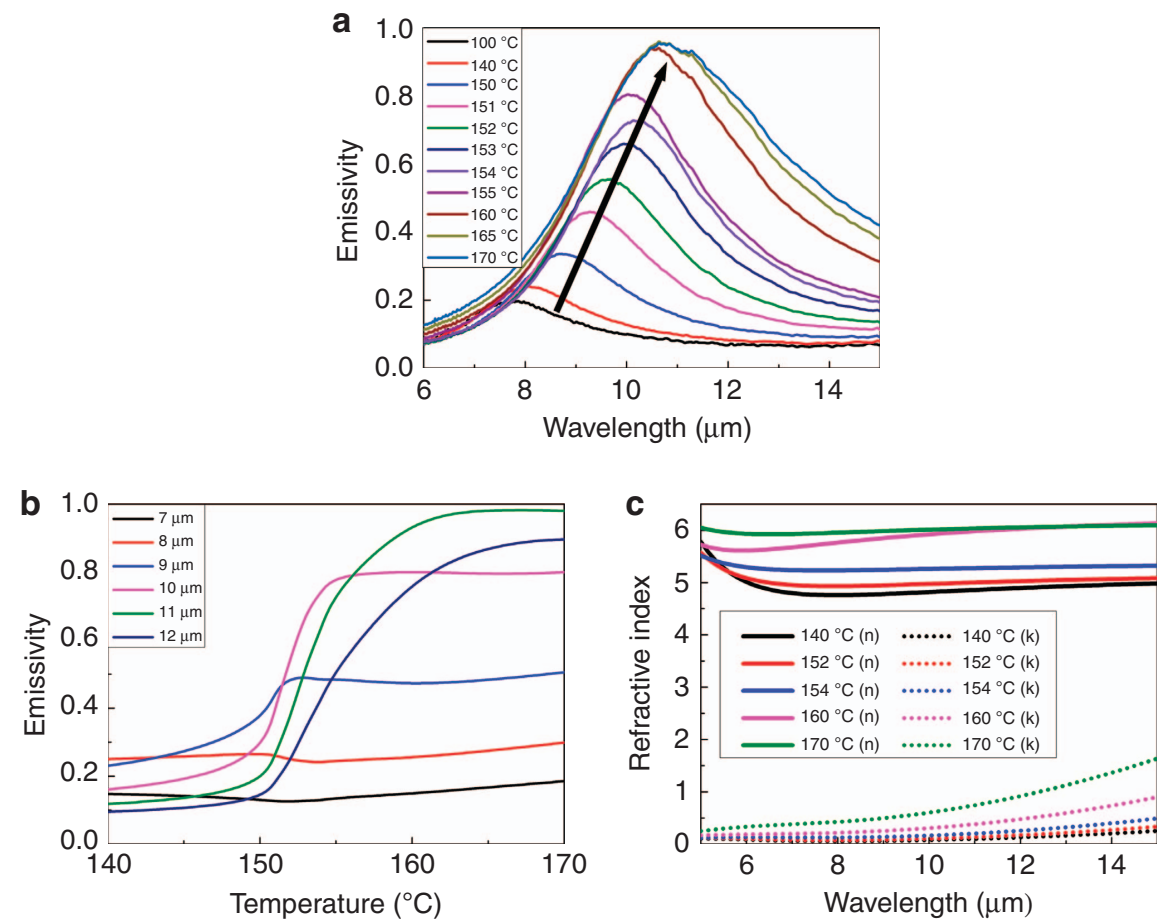

Figure 6 (a) Tunable emissivity of the cGST-Au emitter in the mid-infrared with increasing temperature from 100 to $170{ }^{\circ} \mathrm{C}$. (b) Temperature response of the emissivity for the cGST-Au emitter at several typical wavelengths. (c) Measured refractive indices of the GST at different temperatures. $\mathrm{n}$ and $\mathrm{k}$ denote the real and imaginary parts of the refractive indices, respectively. The GST thickness is $450 \mathrm{~nm}$.

is not applied owing to a low-fusion point of the 120 -nm-thick gold film. The thermal reamorphization of the GST on a silicon wafer at a temperature above $640{ }^{\circ} \mathrm{C}$ is performed, and the infrared properties are presented in Supplementary Fig. S8, demonstrating the reamorphization ability of the cGST. Because the Au film merely plays the role of a PEC, by replacing it with other metal films with higher-fusion points (such as $\mathrm{Ti}, \mathrm{W}$ and $\mathrm{Mo}$ ), this emitter can also be reversely switchable from the on state back to the off state. The switching speed depends on the phase transition speed of the GST. The phase transition process takes several seconds with the thermal annealing method in our experiment. This timescale can be decreased by increasing the annealing temperature (Supplementary Fig. S7) or reducing the thermal substrate effect. In addition to thermal annealing, the crystallization and reamorphization of the GST can also be realized using proper laser pulses with high peak power ${ }^{51,60-63}$ or electrical stimulations ${ }^{47}$. The typical switching time for electrical stimulation is nanoseconds ${ }^{47}$. By stimulating the sample with laser pulses, the switching time can be nanoseconds ${ }^{63}$ or even improved to femtoseconds ${ }^{61}$. So far, the emission property of the GST-Au emitter has been exclusively demonstrated in the $8-14 \mu \mathrm{m}$ atmospheric window. The cGST-Au samples have simultaneous switchable, tunable and wavelength-selective emission properties in the 3-5 $\mu \mathrm{m}$ atmospheric window (shown in Supplementary Fig. S9), demonstrating the capability of dual-band emission in both thermal atmospheric windows.

\section{CONCLUSIONS}

In summary, control over the emissivity of zero-static-power midinfrared thermal emitters consisting of a thermally stable GST layer and a metal layer is demonstrated. The GST film does not require sustained thermal consumption to preserve its phase. The metal film plays the role of a PEC, which not only halves the GST thickness but also significantly enhances the resonant peak emissivity of the emitter. Broad wavelength-selectivity is realized simply by controlling the GST thickness. An emissivity approaching the ideal blackbody maximum is achieved when the GST is in the crystalline phase, and a maximum extinction ratio of above $10 \mathrm{~dB}$ can be obtained between the two states (on state and off state) of the emitter. Moreover, by controlling the intermediate phases composed of different proportions of the amorphous and crystalline molecules of the GST, the thermal emissivity can be continuously tunable. Moreover, because only layered films are involved in the fabrication, this thermal emitter benefits from the merits of large-area and lithography-free fabrication as well as design flexibility. Combining all these advantages, this switchable, tunable, wavelength-selective zero-static-power thermal emitter will pave the way toward the ultimate control of thermal emissivity in the field of fundamental science as well as for energy-harvesting and thermal control applications, including thermophotovoltaics, light sources, infrared imaging and radiative coolers.

\section{CONFLICT OF INTEREST}

The authors declare no conflict of interest.

\section{ACKNOWLEDGEMENTS}

This work is supported by the National Natural Science Foundation of China (grant nos 61425023, 61575177, 61275030 and 61235007).

1 Karker N, Dharmalingam G, Carpenter MA. Thermal energy harvesting plasmonic based chemical sensors. ACS Nano 2014; 8: 10953-10962.

2 Vorobyev AY, Makin VS, Guo CL. Brighter light sources from black metal: significant increase in emission efficiency of incandescent light sources. Phys Rev Lett 2009; 102: 234301.

3 Park JH, Han SE, Nagpal P, Norris DJ. Observation of thermal beaming from tungsten and molybdenum bull's eyes. ACS Photonics 2016; 3: 494-500. 
4 Lenert A, Bierman DM, Nam Y, Chan WR, Celanović I et al. A nanophotonic solar thermophotovoltaic device. Nat Nanotechnol 2014; 9: 126-130.

5 Rephaeli E, Fan SH. Absorber and emitter for solar thermo-photovoltaic systems to achieve efficiency exceeding the Shockley-Queisser limit. Opt Express 2009; 17: 15145-15159.

6 Chan WR, Bermel P, Pilawa-Podgurski RCN, Marton CH, Jensen KF et al. Toward high-energy-density, high-efficiency, and moderate-temperature chip-scale thermophotovoltaics. Proc Natl Acad Sci USA 2013; 110: 5309-5314.

7 Nagpal P, Han SE, Stein A, Norris DJ. Efficient low-temperature thermophotovoltaic emitters from metallic photonic crystals. Nano Lett 2008; 8: 3238-3243.

8 Liu J, Guler U, Li W, Kildishev A, Boltasseva A et al. High-temperature plasmonic thermal emitter for thermo-photovotaics. In: CLEO: QELS_Fundamental Science. San Jose, CA, USA: Optical Society of America; 2014.

9 Raman AP, Anoma MA, Zhu LX, Rephaeli E, Fan SH. Passive radiative cooling below ambient air temperature under direct sunlight. Nature 2014; 515: 540-544.

10 Rephaeli E, Raman A, Fan SH. Ultrabroadband photonic structures to achieve highperformance daytime radiative cooling. Nano Lett 2013; 13: 1457-1461.

11 Zhu LX, Raman AP, Fan SH. Radiative cooling of solar absorbers using a visibly transparent photonic crystal thermal blackbody. Proc Natl Acad Sci USA 2015; 112: 12282-12287.

12 Kats MA, Blanchard R, Zhang SY, Genevet P, Ko C et al. Vanadium dioxide as a natura disordered metamaterial: perfect thermal emission and large broadband negative differential thermal emittance. Phys Rev X 2013; 3: 041004.

13 Franke EB, Trimble CL, Schubert M, Woollam JA, Hale JS. All-solid-state electrochromic reflectance device for emittance modulation in the far-infrared spectral region. Appl Phys Lett 2000; 77: 930-932.

14 Benkahoul M, Chaker M, Margot J, Haddad E, Kruzelecky R et al. Thermochromic VO film deposited on Al with tunable thermal emissivity for space applications. Sol Energy Mater Sol Cells 2011; 95: 3504-3508.

15 Yu ZF, Sergeant NP, Skauli T, Zhang G, Wang HL et al. Enhancing far-field thermal emission with thermal extraction. Nat Commun 2013; 4: 1730.

16 Greffet JJ, Carminati R, Joulain K, Mulet JP, Mainguy S et al. Coherent emission of light by thermal sources. Nature 2002; 416: 61-64.

17 Pralle MU, Moelders N, McNeal MP, Puscasu I, Greenwald AC et al. Photonic crystal enhanced narrow-band infrared emitters. App/ Phys Lett 2002; 81: 4685-4687.

18 Wang Z, Luk TS, Tan YX, Ji DX, Zhou M et al. Tunneling-enabled spectrally selective thermal emitter based on flat metallic films. Appl Phys Lett 2015; 106: 101104

19 Celanovic I, Perreault D, Kassakian J. Resonant-cavity enhanced thermal emission. Phys Rev B 2005; 72: 075127

20 Roberts AS, Chirumamilla M, Thilsing-Hansen K, Pedersen K, Bozhevolnyi SI. Near-infrared tailored thermal emission from wafer-scale continuous-film resonators. Opt Express 2015; 23: A1111-A1119.

21 Schuller JA, Taubner T, Brongersma ML. Optical antenna thermal emitters. Nat Photonics 2009; 3: 658-661.

22 Liu XL, Tyler T, Starr T, Starr AF, Jokerst NM et al. Taming the blackbody with infrared metamaterials as selective thermal emitters. Phys Rev Lett 2011; 107: 045901.

23 Jellison GE, Modine FA. Parameterization of the optical functions of amorphous materials in the interband region. Appl Phys Lett 1996; 69: 371-373.

24 Makhsiyan M, Bouchon P, Jaeck J, Pelouard JL, Haïdar R. Shaping the spatial and spectral emissivity at the diffraction limit. App/ Phys Lett 2015; 107: 251103.

25 Costantini D, Lefebvre A, Coutrot AL, Moldovan-Doyen I, Hugonin JP et al. Plasmonic metasurface for directional and frequency-selective thermal emission. Phys Rev Appl 2015; 4: 014023

26 Streyer W, Feng K, Zhong Y, Hoffman AJ, Wasserman D. Selective absorbers and thermal emitters for far-infrared wavelengths. Appl Phys Lett 2015; 107: 081105.

27 Yeng YX, Ghebrebrhan M, Bermel P, Chan WR, Joannopoulos JD et al. Enabling hightemperature nanophotonics for energy applications. Proc Natl Acad Sci USA 2012; 109: 2280-2285.

28 Arpin KA, Losego MD, Cloud AN, Ning HL, Mallek J et al. Three-dimensional selfassembled photonic crystals with high temperature stability for thermal emission modification. Nat Commun 2013; 4: 2630.

29 Qu YR, Li Q, Gong HM, Du KK, Bai S et al. Spatially and spectrally resolved narrowband optical absorber based on 2D grating nanostructures on metallic films. Adv Opt Mater 2016; 4: 480-486.

30 Manjavacas A, Thongrattanasiri S, Greffet JJ, de Abajo FJG. Graphene optical-tothermal converter. Appl Phys Lett 2014; 105: 211102.

31 Inoue T, Asano T, de Zoysa M, Oskooi A, Noda S. Design of single-mode narrowbandwidth thermal emitters for enhanced infrared light sources. J Opt Soc Am B 2013; 30: $165-172$

32 Inoue T, de Zoysa M, Asano T, Noda S. Filter-free nondispersive infrared sensing using narrow-bandwidth mid-infrared thermal emitters. App/ Phys Express 2014; 7: 012103.

33 Inoue T, de Zoysa M, Asano T, Noda S. Realization of dynamic thermal emission control. Nat Mater 2014; 13: 928-931.

34 de Zoysa M, Asano T, Mochizuki K, Oskooi A, Inoue T et al. Conversion of broadband to narrowband thermal emission through energy recycling. Nat Photonics 2012; 6: 535-539.

35 Inoue T, de Zoysa M, Asano T, Noda S. On-chip integration and high-speed switching of multi-wavelength narrowband thermal emitters. App/ Phys Lett 2016; 108: 091101.

36 Brar VW, Sherrott MC, Jang MS, Kim S, Kim L et al. Electronic modulation of infrared radiation in graphene plasmonic resonators. Nat Commun 2015; 6: 7032
37 Liu XY, Padilla WJ. Thermochromic infrared metamaterials. Adv Mater 2016; 28 871-875.

38 Athanasopoulos N, Siakavellas NJ. Programmable thermal emissivity structures based on bioinspired self-shape materials. Sci Rep 2015; 5: 17682.

39 Tittl A, Michel AKU, Schäferling M, Yin XH, Gholipour B et al. A switchable mid-infrared plasmonic perfect absorber with multispectral thermal imaging capability. Adv Mater 2015; 27: 4597-4603.

40 Yin XH, Schäferling M, Michel AU, Tittl A, Wuttig M et al. Active chiral plasmonics. Nano Lett 2015; 15: 4255-4260.

41 Schlich FF, Zalden P, Lindenberg AM, Spolenak R. Color switching with enhanced optical contrast in ultrathin phase-change materials and semiconductors induced by femtosecond laser pulses. ACS Photonics 2015; 2: 178-182.

42 Kao TS, Chen YG, Hong MH. Controlling the near-field excitation of nano-antennas with phase-change materials. Beilstein J Nanotechnol 2013; 4: 632-637.

43 Michel AU, Chigrin DN, Maß TWW, Schönauer K, Salinga M et al. Using low-loss phase-change materials for mid-infrared antenna resonance tuning. Nano Lett 2013; 13: 3470-3475.

44 Chen YG, Kao TS, Ng B, Li X, Luo XG et al. Hybrid phase-change plasmonic crystals for active tuning of lattice resonances. Opt Express 2013; 21: 13691-13698.

45 Li PN, Yang XS, Maß TWW, Hanss J, Lewin M et al. Reversible optical switching of highly confined phonon-polaritons with an ultrathin phase-change material. Nat Mater 2016; 15: 870-875.

46 Wuttig M, Yamada N. Phase-change materials for rewriteable data storage. Nat Mater 2007: 6: 824-832.

$47 \mathrm{Hu}$ YF, Zou H, Zhang JH, Xue JZ, Sui YX et al. $\mathrm{Ge}_{2} \mathrm{Sb}_{2} \mathrm{Te}_{5} / \mathrm{Sb}$ superlattice-like thin film for high speed phase change memory application. Appl Phys Lett 2015; 107: 263105

48 Kohara S, Kato K, Kimura S, Tanaka H, Usuki T et al. Structural basis for the fast phase change of $\mathrm{Ge}_{2} \mathrm{Sb}_{2} \mathrm{Te}_{5}$ : ring statistics analogy between the crystal and amorphous states. Appl Phys Lett 2006; 89: 201910.

49 Cotton RL, Siegel J. Stimulated crystallization of melt-quenched $\mathrm{Ge}_{2} \mathrm{Sb}_{2} \mathrm{Te}_{5}$ films employing femtosecond laser double pulses. J App/ Phys 2012; 112: 123520.

50 Sittner ER, Siegert KS, Jost P, Schlockermann C, Lange FRL et al. $(\mathrm{GeTe})_{x}-\left(\mathrm{Sb}_{2} \mathrm{Te}_{3}\right)_{1-x}$ phase-change thin films as potential thermoelectric materials. Phys Status Solidi $A$ 2013; 210: 147-152.

51 Ríos C, Stegmaier M, Hosseini P, Wang D, Scherer T et al. Integrated all-photonic nonvolatile multi-level memory. Nat Photonics 2015; 9: 725-732.

52 Hosseini $\mathrm{P}$, Wright $\mathrm{CD}$, Bhaskaran $\mathrm{H}$. An optoelectronic framework enabled by lowdimensional phase-change films. Nature 2014; 511: 206-211.

53 Ríos C, Hosseini P, Taylor RA, Bhaskaran H. Color depth modulation and resolution in phase-change material nanodisplays. Adv Mater 2016; 28: 4720-4726.

54 Rakić AD, Djurišić AB, Elazar JM, Majewski ML. Optical properties of metallic films for vertical-cavity optoelectronic devices. Appl Opt 1998; 37: 5271-5283.

55 Zhu DX, Shen WD, Zhen HY. Anisotropic optical constants of in-plane oriented polyfluorene thin films on rubbed substrate. J Appl Phys 2009; 106: 084504.

56 Botten LC, McPhedran RC, Nicorovici NA, Derrick GH. Periodic models for thin optima absorbers of electromagnetic radiation. Phys Rev B 1997; 55: R16072.

57 Piper JR, Fan SH. Total absorption in a graphene monolayer in the optical regime by critical coupling with a photonic crystal guided resonance. ACS Photonics 2014; 1 : 347-353.

58 Kats MA, Blanchard R, Genevet P, Capasso F. Nanometre optical coatings based on strong interference effects in highly absorbing media. Nat Mater 2012; 12 : 20-24.

59 Hao J, Zhou L, Qiu M. Nearly total absorption of light and heat generation by plasmonic metamaterials. Phys Rev B 2011; 83: 165107.

60 Hira T, Homma T, Uchiyama T, Kuwamura K, Kihara Y et al. All-optical switching of localized surface plasmon resonance in single gold nanosandwich using GeSbTe film as an active medium. Appl Phys Lett 2015; 106: 031105.

61 Michel AU, Zalden P, Chigrin DN, Wuttig M, Lindenberg AM et al. Reversible optical switching of infrared antenna resonances with ultrathin phase-change layers using femtosecond laser pulses. ACS Photonics 2014; 1: 833-839.

62 Rios C, Hosseini P, Wright CD, Bhaskaran H, Pernice WHP. On-chip photonic memory elements employing phase-change materials. Adv Mater 2014; 26: 1372-1377.

63 Gholipour B, Zhang JF, MacDonald KF, Hewak DW, Zheludev NI. An all-optical, non-volatile, bidirectional, phase-change meta-switch. Adv Mater 2013; 25 3050-3054.

(c) (1) (2) This work is licensed under a Creative Commons Attributioncc. ${ }_{\text {BY NC SA }}$ NonCommercial-ShareAlike 4.0 International License. The images or other third party material in this article are included in the article's Creative Commons license, unless indicated otherwise in the credit line; if the material is not included under the Creative Commons license, users will need to obtain permission from the license holder to reproduce the material. To view a copy of this license, visit http:// creativecommons.org/licenses/by-nc-sa/4.0/

(C) The Author(s) 2017

Supplementary Information for this article can be found on the Light: Science \& Applications' website (http://www.nature.com/lsa). 